\title{
Matching Preclusion and Conditional Matching Preclusion for Bipartite Interconnection Networks II: Cayley Graphs Generated by Transposition Trees and Hyper-stars
}

\author{
Eddie Cheng \\ Department of Mathematics and Statistics, Oakland University, Rochester, Michigan 48309
}

Philip Hu

Yale University, New Haven, Connecticut 06511

Roger Jia

University of Michigan, Ann Arbor, Michigan 48109

\author{
László Lipták \\ Department of Mathematics and Statistics, Oakland University, Rochester, Michigan 48309
}

\begin{abstract}
The matching preclusion number of a graph with an even number of vertices is the minimum number of edges whose deletion results in a graph that has no perfect matchings. For many interconnection networks, the optimal sets are precisely those induced by a single vertex. It is natural to look for obstruction sets beyond those induced by a single vertex. The conditional matching preclusion number of a graph is the minimum number of edges whose deletion results in a graph with no isolated vertices that has no perfect matchings. In this companion paper of Cheng et al. (Networks (NET 1554)), we find these numbers for a number of popular interconnection networks including hypercubes, star graphs, Cayley graphs generated by transposition trees and hyper-stars. () 2011 Wiley Periodicals, Inc. NETWORKS, Vol. 59(4), 357-364 2012
\end{abstract}

Keywords: interconnection networks; perfect matching; Cayley graphs; hyper-stars

\section{INTRODUCTION}

Let $G$ be a graph with an even number of vertices. A perfect matching in $G$ is a set of edges such that every vertex is incident with exactly one edge in this set. The matching preclusion number of graph $G$, denoted by $\operatorname{mp}(G)$, is

Received September 2009; accepted February 2011

Correspondence to: L. Lipták; e-mail: liptak@oakland.edu

Contract grant sponsor: NSF-REU; Contract grant number: DMS 0649099 DOI 10.1002/net.20441

Published online 22 April 2011 in Wiley Online Library (wileyonlinelibrary.com).

C) 2011 Wiley Periodicals, Inc. the minimum number of edges whose deletion leaves the resulting graph without a perfect matching. Any such optimal set is called an optimal matching preclusion set. We define $\operatorname{mp}(G)=0$ if $G$ has no perfect matchings. This concept of matching preclusion was introduced by [2] and further studied by $[5,11]$ with special attention given to interconnection networks. The following observation is obvious:

Proposition 1.1. Let $G$ be a graph with an even number of vertices. Then $m p(G) \leq \delta(G)$, where $\delta(G)$ is the minimum degree of $G$.

If $\operatorname{mp}(G)=\delta(G)$, then $G$ is maximally matched. We call an optimal solution consisting of all edges incident to a vertex of minimum degree a trivial optimal matching preclusion set. A graph $G$ is super matched, if $\operatorname{mp}(G)=\delta(G)$ and every optimal matching preclusion set is trivial. In a distributed system, it is unlikely that, in the event of random link failures, all edges at some vertex fail. Hence it is natural to ask what the obstruction sets are for a graph with link failures to have a perfect matching subject to the condition that the faulty graph has no isolated vertices. This motivates the following definition [6]: The conditional matching preclusion number of a graph $G$ with an even number of vertices, denoted by $\mathrm{mp}_{1}(G)$, is the minimum number of edges whose deletion leaves the resulting graph with no isolated vertices and without a perfect matching. Any such optimal set is called an optimal conditional matching preclusion set. We define $\operatorname{mp}_{1}(G)=0$ if $G$ has no perfect matchings, and we will leave $\mathrm{mp}_{1}(G)$ undefined if a conditional matching preclusion 
set does not exist, that is, we cannot delete edges to satisfy both conditions in the definition. Note that if we pick any 2-path $u-v-w$ in the original graph and delete all the edges incident to either $u$ or $w$ but not $v$, then the resulting graph has no perfect matchings. Thus, we define

$$
\begin{aligned}
& v_{e}(G)=\min \left\{d_{G}(u)\right. \\
& \left.+d_{G}(v)-2-y_{G}(u, v): u \text { and } v \text { are ends of a 2-path }\right\},
\end{aligned}
$$

where $d_{G}(u)$ is the degree of vertex $u$ and $y_{G}(u, v)=1$ if $u$ and $v$ are adjacent and 0 otherwise. (We will suppress $G$ and simply write $d$ and $y$ if it is clear from the context.) Then mirroring Proposition 1.1, we have the following easy result:

Proposition 1.2. Let $G$ be a graph with an even number of vertices. Suppose that every vertex in $G$ has degree at least 3. Then $m p_{1}(G) \leq v_{e}(G)$.

If $\operatorname{mp}_{1}(G)=v_{e}(G)$, then $G$ is called conditionally maximally matched. We call an optimal solution of the form induced by $v_{e}$ a trivial optimal conditional matching preclusion set. Since the matching preclusion number measures the robustness in the event of link failures, it is desirable for an interconnection network to be super matched. Similarly, it is desirable to have the property that all optimal conditional matching preclusion sets are trivial as well. Such an interconnection network is conditionally super matched. Ref. [6] introduced this concept and considered the conditional matching preclusion problem for a number of basic networks including hypercubes, and it was proved that they have this desired property.

We use standard graph theory terminology which can be found in West [16]. If the (vertex) connectivity of $G$ is $\delta(G)$, then $G$ is maximally connected; if the edge-connectivity of $G$ is $\delta(G)$, then $G$ is maximally edge-connected. A graph $G$ is tightly super connected if, after the deletion of at most $\delta(G)$ vertices, the graph either remains connected or has exactly two connected components, one of which is a singleton, that is, an isolated vertex. Naturally, these concepts can also apply to deletion of edges. Therefore, we define a maximally edgeconnected graph $G$ to be super edge-connected if the deletion of at most $\delta(G)$ edges results in either a connected graph or exactly two connected components, one of which is a singleton. (We note that unlike the deletion of vertices, here we can replace "deletion of at most $\delta(G)$ edges" by "deletion of $\delta(G)$ edges.")

The above concepts can be generalized further. A graph is super $m$-edge-connected of order $q$ if with at most $m$ edges deleted, the resulting graph is either connected or it has one large component and a number of small components with at most $q$ vertices in total. So a super edge-connected graph $G$ is super $\delta(G)$-edge-connected of order 1. Similarly, a graph is super $m$-connected of order $q$ if with at most $m$ vertices deleted, the resulting graph is either connected or it has one large component and a number of small components with at most $q$ vertices in total.

This article is organized as follows: In the rest of this section, we state the general results that are proved in the companion paper [3] for the matching preclusion and the conditional matching preclusion number as well as the classification of optimal solutions in the respective problems for bipartite graphs. In the subsequent sections, we apply these results to a number of interconnection networks: hypercubes in section 2, star graphs in section 3, Cayley graphs generated by transposition trees in section 4 , and hyper-stars in section 5 .

We start with the following result whose validity follows directly from the well-known fact that the edges of a $k$-regular bipartite graph can be partitioned into $k$ disjoint perfect matchings:

Theorem 1.3. Let $G$ be a k-regular bipartite graph. Then $G$ is maximally matched, that is, $\operatorname{mp}(G)=k$.

The following theorems give sufficient conditions for a regular bipartite graph to be super matched, conditionally maximally matched and conditionally super matched. Note that if $G$ is a $k$-regular bipartite graph, then $v_{e}(G)=2 k-2$.

Theorem 1.4 ([3]). Let $G$ be a k-regular bipartite graph that is super edge-connected. Then $G$ is super matched.

Theorem 1.5 ([3]). Let $G$ be a $k$-regular bipartite graph that is super $(3 k-6)$-edge-connected of order 2 . Then $m p_{1}(G)=$ $2 k-2$.

Theorem 1.6 ([3]). Let $G$ be a k-regular bipartite graph with $m_{1}(G)=2 k-2$. If $G$ is super $(3 k-4)$-edge-connected of order 3 , then it is conditionally super matched.

In the study of structural properties, researchers usually deal with the property of super $m$-connected of order $q$ rather than super $m$-edge-connected of order $q$ as the vertex version is stronger if the graph is large enough. So we state the corresponding vertex version of the sufficient conditions here, as they will be useful in this article.

Corollary 1.7. Let $G$ be a $k$-regular bipartite graph that is tightly super connected. If $G$ has at least $k+4$ vertices, then $G$ is super matched. In particular, if $k \geq 4$, then a tightly super connected $k$-regular bipartite graph is super matched.

Corollary 1.8. Let $k \geq 2$. Suppose $G$ is a k-regular bipartite graph with at least $\max \{3 k, 7\}$ vertices. If $G$ is super $(3 k-$ 6)-connected of order 2 , then $\operatorname{mp}_{1}(G)=2 k-2$.

Corollary 1.9. Let $k \geq 2$. Suppose $G$ is a $k$-regular bipartite graph with $m p_{1}(G)=2 k-2$. If $G$ has at least $3 k+4$ vertices and it is super $(3 k-4)$-connected of order 3 , then it is conditionally super matched.

\section{HYPERCUBES}

In this section, we apply our general results for the first useful and classical interconnection networks, namely, the 
hypercubes. Although the results in the section are known, they serve as useful illustrations of the general results. Let $n \geq 3$. The hypercube $B_{n}$ is the graph in which the vertex set is the set of binary strings of length $n$, and two vertices are adjacent if and only if the two strings differ in exactly one position. Clearly, $B_{n}$ is bipartite with the bipartition sets being the set of strings with an odd number of 1's and the set of strings with an even number of 1's. The theorems that we want to prove are the following:

Theorem 2.1 ([2]). Let $n \geq 2$. Then $m p\left(B_{n}\right)=n$, and $B_{n}$ is super matched.

Theorem 2.2 ([11]). Let $n \geq 3$. Then $m p_{1}\left(B_{n}\right)=2 n-2$, and $B_{n}$ is conditionally super matched.

Hypercubes are well studied and, in particular, researchers are interested in how this graph will break up when many faulty vertices are present. Here we can utilize Corollary 1.7, Corollary 1.8, Corollary 1.9, and the following theorem:

Theorem 2.3 ([17-19]). If $n \geq 3$, then $B_{n}$ is super $(2 n-3)$ connected of order 1 . If $n \geq 5$, then $B_{n}$ is super $(3 n-6)$ connected of order 2 . If $n \geq 6$, then $B_{n}$ is super $(4 n-10)$ connected of order 3.

Note that Ref. [17-19] actually proved a more general result involving the deletion of linearly many vertices. The first statement of Theorem 2.3 and Corollary 1.7 give Theorem 2.1 for $n \geq 3$. The case $n=2$ can be checked separately. The second statement of Theorem 2.3 and Corollary 1.8 give $\operatorname{mp}_{1}\left(B_{n}\right)=2 n-2$ for $n \geq 5$. The cases $n=3,4$ can be checked separately. Since $4 n-10 \geq 3 n-4$ for $n \geq 6$, we may apply the third statement of Theorem 2.3 and Corollary 1.9 to prove Theorem 2.2 for $n \geq 6$. Of course, this still leaves the cases $n=3,4,5$. Clearly, the case $n=3$ can be checked easily. The case $n=4$ can be checked by a computer using a naive brute force approach. For the case $n=5$, one can either apply a modified brute force method or use an $\mathrm{ad}$ hoc method. We omit the details.

One may wonder why there is a gap in the three small cases. The first reason is that the condition in Corollary 1.9 is only sufficient. The second reason is that $B_{n}$ has 4-cycles, so a path of length 2 can be isolated by the deletion of fewer vertices than edges, thus Corollary 1.9 cannot be used for small $n$. Of course, one can prove a sharper result that is the edge version of Theorem 2.3, but we have decided to use only existing results here, as Theorem 2.2 is already known.

\section{STAR GRAPHS}

In this section, we apply our general results to the star graphs, another popular class of interconnection networks introduced in [1] to serve as a competitive model to the class of hypercubes.

Let $n \geq 3$. The star graph $S_{n}$ has the $n$ ! permutations on $\{1,2, \ldots, n\}$ as its vertex set. Two vertices $\left[a_{1}, a_{2}, \ldots, a_{n}\right]$ and

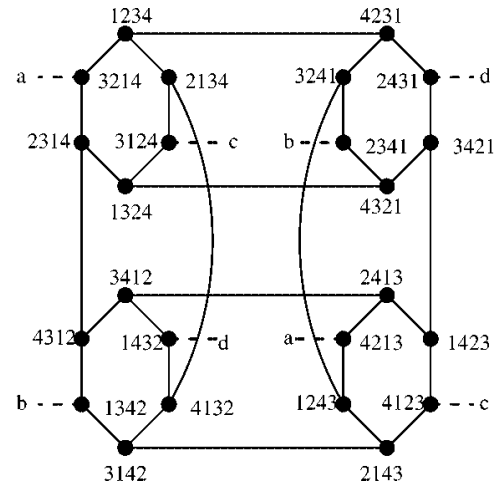

FIG. 1. Star graph $S_{4}$.

$\left[b_{1}, b_{2}, \ldots, b_{n}\right]$ are adjacent if there exists an $i \in\{2,3, \ldots, n\}$ such that $a_{1}=b_{i}, b_{1}=a_{i}$ and $a_{j}=b_{j}$ for all $j \in$ $\{2,3, \ldots, n\}-\{i\}$. In other words, they are adjacent if one can be obtained from the other by interchanging the symbols in position 1 and position $i$ for some $i=2,3, \ldots, n$. So $S_{n}$ is $(n-1)$-regular and bipartite with the set of even permutations and the set of odd permutations as the bipartition sets. It is not difficult to see that $S_{n}$ has girth 6 (that is, the shortest cycle is of length 6), and it is vertex-transitive and edge-transitive. Figure 1 shows $S_{4}$. If convenient, we will write $a_{1} a_{2} \ldots a_{n}$ rather than $\left[a_{1}, a_{2}, \ldots, a_{n}\right]$ to denote a vertex of $S_{n}$.

Theorem 3.1 ([11]). Let $n \geq 3$. Then $m p\left(S_{n}\right)=\delta\left(S_{n}\right)=$ $n-1$. Moreover, if $n \geq 4$, then $S_{n}$ is super matched.

Theorem 3.2. Let $n \geq 3$. Then $m p_{1}\left(S_{n}\right)=2 n-4$ and $S_{n}$ is conditionally super matched.

As in the case of hypercubes, Theorem 3.1 follows from the fact that $S_{n}$ is tightly super connected for $n \geq 4$, as shown in [7], and Corollary 1.7. Theorem 3.2 can be proved using Hamiltonicity results as in [12]. Here we will prove it using the sufficient conditions given in Section 1, but we will delay the proof to the next section where we consider a generalization of star graphs.

\section{CAYLEY GRAPHS GENERATED BY TRANSPOSITION TREES}

In this section, we apply our general results to Cayley graphs generated by transposition trees, which generalize the star graphs.

Let $\Gamma$ be a finite group and let $S$ be a set of elements of $\Gamma$ such that the identity of the group does not belong to $S$. The Cayley graph $\Gamma(S)$ is the directed graph with vertex set $\Gamma$ in which there is an arc from $u$ to $v$ if there is an $s \in S$ such that $v=u s$. The Cayley graph $\Gamma(S)$ is connected if and only if $S$ is a generating set for $\Gamma$. A Cayley graph is always vertex-transitive.

Here, we choose the finite group to be $\Gamma_{n}$, the set of permutations on $\{1,2, \ldots, n\}$, and the generating set $S$ to be a set of transpositions. The vertices of the corresponding Cayley 


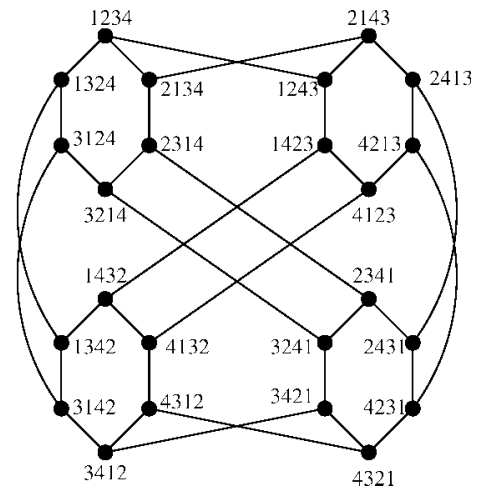

FIG. 2. Bubble-sort graph for $P_{3}$.

graph are permutations, and since $S$ only has transpositions, there is an arc from vertex $u$ to vertex $v$ if and only if there is an $\operatorname{arc}$ from $v$ to $u$. Hence we can regard these Cayley graphs as undirected graphs by replacing each pair of these arcs with an edge. With transpositions as the generating set, a simple way to depict $S$ is via a graph with vertex set $\{1,2, \ldots, n\}$ with an edge between $i$ and $j$ if and only if the transposition ( $i j)$ belongs to $S$. This graph is called the transposition generating graph of $\Gamma_{n}(S)$ or simply its generating graph. In fact, the star graphs were introduced via the generating graph $K_{1, n-1}$, where the center is 1 and the leaves are $2,3, \ldots, n$. We only consider the case when the generating graph is a tree, which we refer to as the transposition generating tree of $\Gamma_{n}(S)$ or simply its generating tree. When $n=3$, the transposition generating tree must be $K_{1,2}$, which generates a 6-cycle. If $n=4$, then it can be $K_{1,3}$ or $P_{3}$, which generate the star graph $S_{4}$ given in Figure 1 and the bubble-sort graph given in Figure 2, respectively. It is clear that a Cayley graph obtained from a transposition generating tree on $\{1,2, \ldots, n\}$ is $(n-1)$ regular and bipartite with the bipartition sets being the set of even permutations and the set of odd permutations. Our goal is to establish the following results:

Theorem 4.1. Let $G$ be a Cayley graph obtained from a transposition generating tree on $\{1,2, \ldots, n\}$ with $n \geq 3$. Then $\operatorname{mp}(G)=\delta(G)=n-1$. Moreover, if $n \geq 4$, then $G$ is super matched.

Theorem 4.2. Let $G$ be a Cayley graph obtained from a transposition generating tree on $\{1,2, \ldots, n\}$ with $n \geq 7$. Then $\operatorname{mp}_{1}(G)=2 n-4$, and $G$ is conditionally super matched.

As in the previous sections, we use existing results on Cayley graphs obtained from transposition generating trees to prove Theorem 4.1 and Theorem 4.2:

Theorem 4.3 ([8]). Let $G$ be a Cayley graph obtained from a transposition generating tree on $\{1,2, \ldots, n\}$ with $n \geq 4$. Then $G$ is tightly super connected.

Theorem 4.4 ([10]). Let $G$ be a Cayley graph obtained from a transposition generating tree on $\{1,2, \ldots, n\}$ with $n \geq 3$.
If $T$ is a set of vertices of $G$ such that $|T| \leq k(n-1)-$ $\frac{k(k+1)}{2}$ where $1 \leq k \leq n-2$, then $G-T$ has one large (connected) component, and the remaining small components have at most $k-1$ vertices in total. (This includes the case when $G-T$ is connected.)

Theorem 4.1 now follows directly from Theorem 4.3 and Corollary 1.7. To prove $\operatorname{mp}_{1}(G)=2 n-4$, it suffices to show that it is super $3(n-1)-6$ connected of order 2 by using Corollary 1.8. This is precisely the condition given in Theorem 4.4 with $k=3$. To be precise, we need $\max \{3(n-$ $1), 7\} \leq n$ !, which is true for $n \geq 4$. The case $n=3$ can be checked by hand as it is just a 6-cycle. To complete the proof of Theorem 4.2, we apply Theorem 4.4 with $k=4$. So $G$ is super $(4 n-14)$-connected of order 3 . Since $4 n-14 \geq 3 n-$ $7=3(n-1)-4$ for $n \geq 7$, we have proved Theorem 4.2 by using Corollary 1.9. We note that Theorem 4.2 is also valid for $n=4,5,6$ (except for the graph in Fig. 2), but Theorem 4.4 is not sufficient to prove it, because it is an asymptotic result. A more precise statement for super connectedness of order 3 is given in [10], and Ref. [4] extends the result of Theorem 4.2 to the cases $n=4,5,6$ except for the graph in Figure 2 .

\section{HYPER-STARS}

In this section, we apply our general results to the hyperstar, which was introduced as a competitive model to address the scalability issue of the star graphs $[14,15]$.

The hyper-star $H S(n, k)$ with $1 \leq k \leq n-1$ is defined as follows: its vertex set is the set of $\{0,1\}$-strings of length $n$ with exactly $k 1$ 's, and two vertices are adjacent if and only if one can be obtained from the other by exchanging the first symbol with a different symbol ( 1 with 0 , or 0 with 1 ) in another position. Hence it is a bipartite graph with bipartition sets $V_{0}(n, k)$ and $V_{1}(n, k)$, where $V_{0}(n, k)\left(V_{1}(n, k)\right.$, respectively) is the set of vertices of $H S(n, k)$ with 0 (1, respectively) in the first position. We may use $V_{0}$ and $V_{1}$ instead of $V_{0}(n, k)$ and $V_{1}(n, k)$ if it is clear from the context. Clearly $H S(n, k)$ has $\left(\begin{array}{l}n \\ k\end{array}\right)$ vertices, every vertex in $V_{0}$ has degree $k$, and every vertex in $V_{1}$ has degree $n-k$. So $H S(n, k)$ is regular if and only if $n=2 k$. Figure 3 shows $H S(6,3)$. Note that it is "interconnected" between a graph isomorphic to $H S(5,2)$

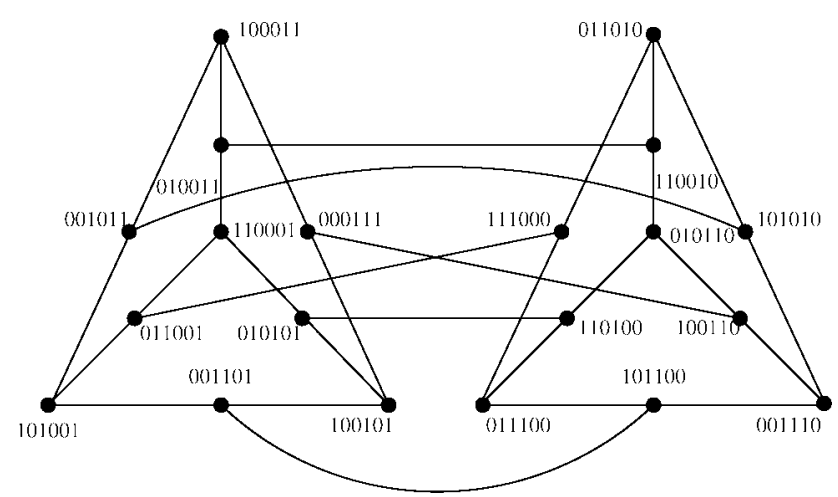

FIG. 3. $H S(6,3)$. 
(the left subgraph whose vertices have 1 in the 6 th position) and a graph isomorphic to $H S(5,3)$ (the right subgraph whose vertices have 0 in the 6 th position). In general $H S(n, k)$ can be decomposed into two graphs: $G_{0}$, the subgraph of $H S(n, k)$ induced by vertices with 0 in the last position, and $G_{1}$, the subgraph of $H S(n, k)$ induced by vertices with 1 in the last position. Clearly $G_{0}$ is isomorphic to $H S(n-1, k)$, and $G_{1}$ is isomorphic to $H S(n-1, k-1)$. The edges between $G_{0}$ and $G_{1}$ are called cross edges. The graphs $H S(n, k)$ and $H S(n, n-k)$ are obviously isomorphic, so we may assume that $n-k \geq k$. The following was proved in [9]:

Theorem 5.1 ([9]). Let $k, n-k \geq 2$. Then HS( $n, k)$ is tightly super connected for $n \geq 5$.

Thus the following result is a consequence of Theorem 5.1 and Corollary 1.7. We note that previous methods using Hamiltonicity results cannot be applied to the hyper-star because it is an open question whether or not $H S(2 k, k)$ is Hamiltonian. (Indeed, this is the famous "middle cube problem.’)

\section{Theorem 5.2. $H S(2 k, k)$ is super matched for $k \geq 2$.}

We remark that the idea of precluding perfect matchings can be extended to precluding saturated matchings for bipartite graphs of unequal bipartition sizes. Indeed, Ref. [3] gives a general result that is applicable to $H S(n, k)$, but we omit the details.

Our next goal is to prove that $H S(2 k, k)$ is conditionally super matched. First, we establish that $H S(2 k, k)$ is conditionally maximally matched by checking that it is super $(3 k-6)$-edge-connected of order 2 . For the classification of optimal conditional matching preclusion sets, we will check that it is super $(3 k-4)$-edge-connected of order 3 . It has been proved in [13] that $H S(n, k)$ is super $(2 \min \{k, n-k\}-2)$ connected of order 2 . However, this is not enough. We need a series of lemmas to prove that $H S(2 k, k)$ is super $(3 k-6)$-edge-connected of order 2 .

Lemma 5.3. Let $n-k \geq k \geq 2$. If we delete at most $3 k-6$ edges in $H S(n, k)$, then there cannot be any components of cardinality 3 in the resulting graph.

Proof. Suppose by contradiction that there is a component of cardinality 3 after the deletion of $3 k-6$ edges. Since there are no odd cycles in the hyper-star, the component must be a path $u-v-w$. Thus all the edges incident to $u, v$, or $w$ must have been deleted except $(u, v)$ and $(v, w)$. Hence there must have been at least $3 k-4$ edges deleted, which is a contradiction.

Obviously the "-6" in Lemma 5.3 is chosen because we want to prove that $H S(n, k)$ is super $(3 k-6)$-edge-connected of order 2 . We need several more lemmas of this type in order for us to prove this result. However, we also need to prove that $H S(n, k)$ is super $(3 k-4)$-edge-connected of order 3.
So in the subsequent lemmas we will consider the deletion of $(3 k-4)$ edges.

Lemma 5.4. Let $n-k \geq k \geq 3$. If we delete at most $3 k-4$ edges in $H S(n, k)$, and there is a component of cardinality 3 in the resulting graph, then the resulting graph has two components, one of which is a path of length 2 ; moreover, $k=n-k$.

Proof. If there is a component of cardinality 3 , then it must be a path $u-v-w$ of length 2 since there are no odd cycles in the hyper-star. From the proof of Lemma 5.3, we get that all the edges incident to $u, v$, and $w$ except $(u, v)$ and $(v, w)$ are deleted. So we have deleted at least $k-1$ edges incident to $u, k-2$ edges incident to $v$ and $k-1$ edges incident to $w$ for a total of at least $3 k-4$ edges. But this means we have deleted exactly $k-1$ edges incident to $u, k-2$ edges incident to $v$ and $k-1$ edges incident to $w$. This can only happen if $k=n-k$, otherwise at least one of the vertices have degree at least $k+1$. We are done if we can show that the graph $H$ obtained by the deletion of $u, v$, and $w$ in $H S(n, k)$ is connected. This is clearly true if $n-k=k \geq 4$, as $H S(n, k)$ has connectivity $k$. If $k=3$, then $n=6$. Let $y$ and $z$ be two distinct vertices in $H$. Then there are three internally vertex disjoint paths between $y$ and $z$ in $H S(6,3)$. Since the degree of $v$ is 3 and $v$ is adjacent to both $u$ and $w$, then if $v$ is on one of these paths, then either $u$ or $w$ must be on the same path. Hence at least one of the paths remains in $H$, so $H$ is connected.

Lemma 5.5. Let $n-k \geq k \geq 3$. If we delete at most $3 k-4$ edges in $H S(n, k)$, then there cannot be any components of cardinality 4 or 5 in the resulting graph.

Proof. Suppose by contradiction that there is a component $Y$ of cardinality 4 or 5 after the deletion of at most $3 k-4$ edges in $H S(n, k)$. Let $a$ be the number of edges in $H S(n, k)$ with exactly one endpoint in $Y$. We may assume that $Y$ is an induced subgraph. Since $H S(n, k)$ has no cycles of length less than $6, Y$ is a tree. So the sum of the degrees of vertices in $Y$ is $2(p-1)$ where $p \in\{4,5\}$. So $a \geq k p-2(p-1)$. But $k p-2 p+2>3 k-4$ for $p=4,5$, which is a contradiction.

Lemma 5.6. Let $n-k \geq k \geq 3$. If we delete at most $3 k-4$ edges in $H S(n, k)$ and there exists a component of cardinality 2 in the resulting graph, then there remain no other components of cardinality less than or equal to 2.

Proof. As before, we can calculate that at least $2 k-2$ edges must be deleted to have a component of cardinality 2 . Suppose that there is another component of cardinality 2 . We can again count $2 k-2$ edges that must be deleted to isolate this component. Furthermore, since there are no cycles of length less than 6 in the hyper-star, we are counting at most one edge twice. Thus to isolate two components of cardinality 2 , we must delete at least $4 k-5>3 k-4$ edges.

Now suppose that there is a singleton in addition to a component of cardinality 2 . Again, we must delete $k$ edges to 
isolate the singleton and $2 k-2$ edges to isolate the component of cardinality 2 . Since there are no cycles of length 3 in the hyper-star, we are counting at most one edge twice, so we must delete at least $3 k-3$ edges to isolate these two components, so we get a contradiction in both cases.

Lemma 5.7. Let $n-k \geq k \geq 3$. If we delete at most $3 k-4$ edges in $H S(n, k)$, then every component of cardinality at least 6 in the resulting graph must have at least three vertices in $V_{0}$ and at least three vertices in $V_{1}$.

Proof. Suppose by contradiction that a component of cardinality 6 or more has two or fewer vertices that belong to $V_{0}$. Then at least four vertices in this component must belong to $V_{1}$. Since $V_{1}$ and $V_{0}$ are the two bipartition sets of $H S(n, k)$, none of the vertices in $V_{1}$ are adjacent. In addition, two vertices in $V_{0}$ have at most one common neighbor, otherwise we get a 4-cycle. Since each vertex in $V_{1}$ has degree at most 2 in this component and at most one of them has degree 2, we must have deleted at least $(k-2)+3(k-1)=$ $4 k-5$ edges. But $4 k-5>3 k-4$, which is a contradiction. The same argument applies to the case when the component has two or fewer vertices belonging to $V_{1}$.

Lemma 5.8. Let $n-k \geq k \geq 3$. If we delete at most $3 k-4$ edges in $H S(n, k)$, then there cannot be more than two singletons in the resulting graph.

Proof. Suppose by contradiction that there are three or more singletons. We need to delete $k$ edges to isolate each of three singletons. In addition, since there are no odd cycles, we are counting at most two edges twice. Thus at least $3 k-2$ edges must be deleted to produce three or more singletons, which is a contradiction.

Theorem 5.9. Let $n-k \geq k \geq 2$. Then $H S(n, k)$ is super $(3 k-6)$-edge-connected of order 2 .

Proof. We will proceed with induction on $n$. Our base case is all $H S(n, k)$ with $n-k \geq k=2$, for which the claim is true since we do not delete any vertices.

Now consider $H S(n, k)$ where $n \geq 6$ and $n-k \geq k \geq$ 3 . We want to show that $H S(n, k)$ is super $(3 k-6)$-edgeconnected. Consider the subgraphs $G_{0}$ and $G_{1}$ induced by the vertices that end in 0 and 1 , respectively. Since $G_{0}$ is isomorphic to $H S(n-1, k)$ and $G_{1}$ is isomorphic to $H S(n-$ $1, k-1)$, we have $\delta\left(G_{0}\right) \geq k-1$ and $\delta\left(G_{1}\right)=k-1$. By our induction hypothesis, both $G_{0}$ and $G_{1}$ are super $(3 k-9)$ edge-connected of order 2 . Suppose we delete an edge set $F$ of cardinality $3 k-6$ from $H S(n, k)$. Let $M=\max \{\mid F \cap$ $\left.E\left(G_{0}\right)||, F \cap E\left(G_{1}\right) \mid\right\}$, and let $G^{S}$ be a $G_{i}$ that gives $M$, that is, $M=\left|F \cap E\left(G^{S}\right)\right|$ and let $G^{T}$ be the other one. We consider the following cases.

CASE 1. $|M| \geq 3 k-7$.

Then we are deleting at most one edge that does not belong to $G^{S}$. By Lemma 5.3, Lemma 5.5, and Lemma 5.7, every component of $G^{S}-F$ that is not a singleton or a $K_{2}$ has at least three vertices in $V_{0}$ and at least three vertices in $V_{1}$. Hence each of these components has at least three neighbors in $G^{T}$ in the graph $H S(n, k)$. But we are deleting at most one edge that does not belong to $G^{S}$. So each of these components has a neighbor in $G^{T}$ in the graph $H S(n, k)-F$. Since we are deleting at most one edge in $G^{T}$ and $G^{T}$ is at least 2-edgeconnected, $G^{T}-F$ remains connected. Therefore the only small components in $H S(n, k)-F$ are singletons and $K_{2}$ 's. By Lemma 5.6, a singleton component and a $K_{2}$ component cannot occur at the same time. In addition, we have at most two singletons by Lemma 5.8, hence we are done.

CASE 2. $|M|=3 k-8$.

Then we are deleting exactly two edges that do not belong to $G^{S}$. By Lemma 5.3, Lemma 5.5, and Lemma 5.7, every component in $G^{S}-F$ that is not a singleton or a $K_{2}$ has at least three vertices in $V_{0}$ and at least three vertices in $V_{1}$. Hence each of these components of $G^{S}-F$ has at least three neighbors in $G^{T}-F$ in $H S(n, k)$. But at most two cross edges are in $F$, hence each of these components of $G^{S}-F$ has a neighbor in $G^{T}-F$ in $H S(n, k)-F$. If $k \geq 4$, then $G^{T}$ is at least 3-edge-connected, so $G^{T}-F$ remains connected and the proof follows as in Case 1 . If $k=3$, then $3 k-8=1$, so we are deleting one edge from $G^{S}$. In addition, the number of edges we delete from $G^{T}$ is at most 1 by definition. Hence both $G^{S}-F$ and $G^{T}-F$ remain connected. There are $\left(\begin{array}{l}n-2 \\ k-1\end{array}\right)=$ $\left(\begin{array}{c}n-2 \\ 2\end{array}\right)$ edges between $G^{T}$ and $G^{S}$. Since $n \geq 6,\left(\begin{array}{c}n-2 \\ 2\end{array}\right) \geq 6$. We are deleting at most two of these cross edges, so $H S(n, k)-F$ remains connected.

CASE 3. $|M|=3 k-9$.

Then exactly three edges in $F$ do not belong to $G^{S}$. If $k \geq 5$, then $G^{T}$ is at least 4-edge-connected, thus $G^{T}-F$ remains connected in $H S(n, k)-F$. By our induction hypothesis, $G^{S}$ is super $(3 k-9)$-edge-connected of order 2 . The component in $G^{S}-F$ that is not a singleton or a $K_{2}$ will have at least $\left(\begin{array}{l}n-2 \\ k-1\end{array}\right)-2$ neighbors in $G^{T}$ in $H S(n, k)$. Since $n-k, k \geq 5$, we have $\left(\begin{array}{l}n-2 \\ k-1\end{array}\right)-2>3$, so there will be an edge between such a component in $G^{S}-F$ and $G^{T}-F$ in $H S(n, k)-F$. Hence $H S(n, k)-F$ has a large component and at most two singleton components or a $K_{2}$.

If $k=4$, then $|M|=3 k-9=3$. By definition, we are deleting exactly three edges in $G^{S}$ and at most three edges in $G^{T}$. Since $G^{T}$ and $G^{S}$ are super edge-connected, both $G^{S}-F$ and $G^{T}-F$ either remain connected or consist of exactly two components, one of which is a singleton. Because $n-k \geq 4$ and $k=4$, there are at least $\left(\begin{array}{l}8-2 \\ 4-1\end{array}\right)=20$ cross edges. At most three cross edges are deleted, so there is an edge between the nonsingleton component in $G^{S}-F$ and the nonsingleton component in $G^{T}-F$ in $H S(n, k)-F$. Hence $H S(n, k)-F$ has a large component and at most two singleton components or a $K_{2}$.

If $k=3$, then $|M|=3 k-9=0$. Thus, by definition, $E\left(G_{0}\right) \cap F$ and $E\left(G_{1}\right) \cap F$ are both empty, so exactly three 
cross edges must have been deleted. Again, there are at least six cross edges, so $H S(n, k)-F$ remains connected.

CASE 4. $|M|<3 k-9$.

Then we are deleting fewer than $3 k-9$ edges from both $G^{S}$ and $G^{T}$. Since $G^{S}$ and $G^{T}$ are super $(3 k-9)$-edge-connected of order 2 by our induction hypothesis, each of $G^{S}-F$ and $G^{T}-F$ is either connected or consists of two components, one of which has at most two vertices, or consists of three components, two of which are singletons. Let $C^{S}$ and $C^{T}$ be the largest components in $G^{S}-F$ and $G^{T}-F$, respectively. There are at least $\left(\begin{array}{l}n-2 \\ k-1\end{array}\right)-4$ edges between $C^{S}$ and $C^{T}$ in $H S(n, k)$. If $k=3$, then $|M|<3 k-9=0$, which is impossible, therefore $k \geq 4$. Note that $\left(\begin{array}{l}n-2 \\ k-1\end{array}\right)-4>3 k-6$ since $n \geq 2 k$. Hence there is at least one edge between $C^{S}$ and $C^{T}$ in $H S(n, k)-F$. Therefore, $H S(n, k)-F$ has one large component and a number of small components with at most four vertices in total. Now apply Lemma 5.3, Lemma 5.5, Lemma 5.6, and Lemma 5.8 to complete the proof.

Therefore, we have the following result.

Theorem 5.10. Let $k \geq 2$. Then $m p_{1}(H S(2 k, k))=2 k-2$.

Proof. Apply Theorem 1.5 and Theorem 5.9.

We have solved the problem of determining $\operatorname{mp}_{1}(H S(2 k, k))$. We will now classify the optimal solutions. This requires the following two lemmas, which are extensions of Lemma 5.5 and Lemma 5.7, respectively.

Lemma 5.11. Let $n-k \geq k \geq 3$. If we delete at most $3 k-4$ edges in $H S(n, k)$, then there cannot be any components of cardinality $6,7,8$, or 9 in the resulting graph.

Proof. Suppose by contradiction that there is a component $Y$ of cardinality 6,7,8, or 9 after the deletion of at most $3 k-4$ edges in $H S(n, k)$. Let $a$ be the number of edges in $H S(n, k)$ with exactly one endpoint in $Y$. We may assume that $Y$ is an induced subgraph. Let $p$ be the number of vertices in $Y$, so $p \in\{6,7,8,9\}$. If $Y$ is a tree, then the sum of the degrees of vertices in $Y$ is $2(p-1)$, so $a \geq k p-2(p-1)$. If $Y$ has a cycle, then it must have exactly one cycle, as the shortest cycle in $H S(n, k)$ is a 6-cycle and $p \leq 9$. Hence the sum of the degrees of vertices in $Y$ is $2(p-1)+2$, so $a \geq k p-2(p-1)-2=k p-2 p$. So in either case $a \geq k p-2 p>3 k-4$ since $p \in\{6,7,8,9\}$, which is a contradiction.

Lemma 5.12. Let $n-k \geq k \geq 3$. If we delete at most $3 k-4$ edges in $H S(n, k)$, then every component of cardinality at least 10 in the resulting graph must have at least five vertices in $V_{0}$ and at least five vertices in $V_{1}$.

Proof. Suppose by contradiction that at most four vertices of a component $T$ of cardinality at least 10 belong to one bipartition set after the deletion of at most $3 k-4$ edges in $H S(n, k)$. Let $n_{0}$ and $n_{1}$ be the number of vertices that belong to $V(T) \cap V_{0}$ and $V(T) \cap V_{1}$, respectively. Without loss of generality, let $n_{0} \leq 4$, so $n_{1} \geq 6$. Label the vertices in $V(T) \cap V_{0}$ by $v_{1}, v_{2}, \ldots, v_{n_{0}}$. Any two vertices in $V(T) \cap V_{0}$ can share at most one common neighbor since there are no 4-cycles in the hyper-star. Hence

$$
\sum_{i=1}^{n_{0}} N_{T}\left(v_{i}\right) \leq n_{1}+\left(\begin{array}{c}
n_{0} \\
2
\end{array}\right) \leq n_{1}+6 .
$$

Therefore, the total number of edges in $T$ is at most $n_{1}+6$. To isolate $T$, at least $k n_{1}-\left(n_{1}+6\right)=(k-1) n_{1}-6$ edges must be deleted. Since $n_{1} \geq 6$, at least $6 k-12$ edges must be deleted. But $6 k-12>3 k-4$ for $k \geq 3$, so we have a contradiction.

Theorem 5.13. Let $n-k \geq k \geq 6$. Then HS(n, $k)$ is super $(3 k-4)$-edge-connected of order 3 .

Proof. From Theorem 5.9, we know that $G_{0}$ and $G_{1}$ are super $(3(k-1)-6)$-edge-connected of order 2 . Let $F$ be an edge set of cardinality $3 k-4$, and let $F^{0}=F \cap E\left(G_{0}\right)$ and $F^{1}=F \cap E\left(G_{1}\right)$. Consider the following cases.

CASE 1. $\left|F^{0}\right| \leq 3 k-9$ and $\left|F^{1}\right| \leq 3 k-9$.

Since $G_{0}$ and $G_{1}$ are super $(3 k-9)$-edge-connected of order 2 and $\left(\begin{array}{l}n-2 \\ k-1\end{array}\right)-4>3 k-4$, the large component in $G_{0}-F^{0}$ and the large component in $G_{1}-F^{1}$ have a cross edge between them in $H S(n, k)-F$. So $H S(n, k)$ is super $(3 k-4)$-edge-connected of order 4 . To reduce the "order 4" to “order 3," we apply Lemma 5.4, Lemma 5.5, Lemma 5.6, and Lemma 5.8.

CASE 2. $3 k-8 \leq\left|F^{0}\right|$ or $3 k-8 \leq\left|F^{1}\right|$.

Suppose $3 k-8 \leq\left|F^{S}\right|$ where $S \in\{0,1\}$, and $F^{T}$ is the other set, so $\left|F^{T}\right| \leq 4$. Since $k, n-k>5$, we know that $G_{T}-F$ remains connected in $H S(n, k)-F$. Let $Y$ be the component in $H S(n, k)-F$ containing $G^{T}-F$. By Lemma 5.5, there are no components of cardinality 4 or 5 in $H S(n, k)-F$. In addition, by Lemma 5.11, there are no components of cardinality $6,7,8$, or 9 in $H S(n, k)-F$. We claim that $Y$ is the only component in $H S(n, k)-F$ of cardinality at least 10 . Suppose not, let $K$ be another such component in $H S(n, k)-F$. Then $K$ must be a component in $G_{S}-F$. By Lemma 5.12, $K$ has at least five vertices in $V_{0}$ and at least five vertices in $V_{1}$. But there are at most four cross edges in $F$, so $K$ is part of $Y$, which is a contradiction. Now by Lemma 5.4, Lemma 5.6, and Lemma 5.8, the rest of the components cannot have more than three vertices in total, therefore we are done.

This establishes our main result. We note that the statement excludes $k \leq 5$, because the proof of Theorem 5.13 requires $k \geq 6$. (For example, we need this assumption to conclude that $G_{T}-F$ remains connected in $H S(n, k)-F$.) 
Like our proof for Cayley graphs generated by transposition trees, covering $k \leq 5$ requires an ad hoc checking for these "small cases." We omit them in this article.

Theorem 5.14. For $k \geq 6, H S(2 k, k)$ is conditionally super matched.

Proof. This follows from Theorem 5.10, Theorem 1.6, and Theorem 5.13.

\section{CONCLUSION}

In this article, we used the general theory developed in [3] to solve the matching preclusion and conditional matching preclusion problems for hypercubes, star graphs, and their generalizations, Cayley graphs generated by transposition trees and hyper-stars.

\section{Acknowledgments}

We thank the anonymous referee for a number of helpful comments and suggestions including the recommendation that the manuscript be divided into two smaller papers.

\section{REFERENCES}

[1] S. Akers, D. Harel, and B. Krishnamurthy, "The star graph: An attractive alternative to the $n$-cube," Proc. Int'l Conf. Parallel Processing, University Park, PA, USA, 1987, pp. 393-400.

[2] R. Brigham, F. Harary, E. Violin, and J. Yellen, Perfectmatching preclusion, Congress Numer 174 (2005), 185-192.

[3] E. Cheng, P. Hu, R. Jia, and L. Lipták, Matching preclusion and conditional matching preclusion for bipartite interconnection networks I: Sufficient conditions, Networks, (NET 1554 will appear at the same time as this paper).

[4] E. Cheng, P. Hu, R. Jia, and L. Lipták, Matching preclusion and conditional matching preclusion for bipartite interconnection networks, Technical Report, Technical Reports Series 2009-2, Oakland University, 2009.
[5] E. Cheng, L. Lesniak, M. Lipman, and L. Lipták, Matching preclusion for alternating group graphs and their generalizations, Int J Found Comput Sci 19 (2008), 1413-1437.

[6] E. Cheng, L. Lesniak, M. Lipman, and L. Lipták, Conditional matching preclusion sets, Inf Sci 179 (2009), 1092-1101.

[7] E. Cheng and M. Lipman, Increasing the connectivity of the star graphs, Networks 40 (2002), 165-169.

[8] E. Cheng and L. Lipták, Structural properties of Cayley graphs generated by transposition trees, Congress Numer 180 (2006), 81-96.

[9] E. Cheng and L. Lipták, Structural properties of hyper-stars, Ars Combin 80 (2006), 65-73.

[10] E. Cheng and L. Lipták, Linearly many faults in Cayley graphs generated by transposition trees, Info Sci 177 (2007), 4877-4882.

[11] E. Cheng and L. Lipták, Matching preclusion for some interconnection networks, Networks 50 (2007), 173-180.

[12] E. Cheng, L. Lipták, L.H. Hsu, J. Tan, and C.K. Lin, Conditional matching preclusion for the star graphs, Ars Combin, (in press).

[13] E. Cheng and M. Shah, A strong structural theorem for hyperstars, Congress Numer 80 (2006), 65-73.

[14] J. Kim, E. Oh, H. Lim, and T. Heo, "Topological and communication aspects of hyper-star graphs," Lecture Notes in Comput. Sci. 2869, Springer, Berlin, 2003, pp. 51-58.

[15] H. Lee, J. Kim, E. Oh, and H. Lim, "Hyper-star graph: A new interconnection network improving the network cost of the hypercube," Lecture Notes in Comput. Sci. 2510, Springer, Berlin, 2002, pp. 858-865.

[16] D. West, Introduction to graph theory, Prentice Hall, Upper Saddle River, NY, 1996.

[17] X. Yang, D. Evans, B. Chen, G. Megson, and H. Lai, On the maximal connected component of hypercube with faulty vertices, Int J Comput Math 81 (2004), 515-525.

[18] X. Yang, D. Evans, and G. Megson, On the maximal connected component of hypercube with faulty vertices II, Int J Comput Math 81 (2004), 1175-1185.

[19] X. Yang, D. Evans, and G. Megson, On the maximal connected component of hypercube with faulty vertices III, Int J Comput Math 83 (2006), 27-37. 